\title{
Der offene Zugang als Zukunft der juristischen Informationsversorgung? Das Projekt Swiss Open Law Online (SOL-ONline)
}

\author{
Bernhard Dengg*
}

Schon seit einiger Zeit wird unter VertreterInnen aus Schweizer Rechtsbibliotheken eine rege Diskussion geführt, wie zukünftig Open Access-Inhalte am besten für die Öffentlichkeit angeboten werden können. Im Herbst 2018 startete die UB Bern mit Swiss Open Law Online (SOL-ONline) ein Projekt, das anhand einer Online-Plattform aufzeigen soll, wie juristische Publikationen, aber auch Metadaten, frei zugänglich gemacht werden können. Ziel des Projektes ist nicht, eine funktionierende Datenbank anbieten zu können, sondern aufzuzeigen, in welche Richtung eine zukünftige Informationsversorgung in den Rechtswissenschaften gehen könnte. Das Angebot soll allen publizierenden und interessierten JuristInnen offen stehen.

Die ersten Pflöcke für einen Paradigmenwechsel im wissenschaftlichen Publizieren sind bereits eingeschlagen: Der Schweizerische Nationalfonds (SNF) verlangt, dass die vom SNF geförderten Projekte frei zugänglich publiziert werden. Diesen OpenAccess-Ansatz verfolgt auch die nationale Open-Access-Strategie, die anstrebt, dass bis 2024 alle mit öffentlichen Mitteln geförderte Publikationen frei zugänglich sein sollen.

Diese Zielsetzungen sehen zwar vor, dass Open Access publiziert werden muss, bleiben aber vage bei der Frage, wo überhaupt die Möglichkeit für die Forschenden besteht, Open Access zu publizieren. Gerade in den Rechtswissenschaften fehlt es an entsprechenden Publikationsplattformen, die die Aufschaltung der zu erwartenden Fülle an Publikationen bewältigen könnten. Zwar gibt es bereits Zeitschriften wie sui generis, die den Open Access-Ansatz in sehr professioneller Weise umsetzen, doch basieren diese Ansätze noch auf dem Modell von Online-Zeitschriften. Zukünftig werden jedoch Plattformen gefragt sein, die auf Datenbankebene funktionieren und ein umfassendes Fachspektrum bewältigen können.

Auch die an den Universitäten bereits betriebenen Repositorien sind nicht so konstruiert, dass ihre Inhalte einem Fachpublikum gegenüber aufgearbeitet und eine institutionenübergreifende Recherche erlauben. Vielmehr sind sie institutionell auf die jeweilige Universität oder Fachhochschule ausgerichtet, auch wenn ihre Datenmenge als Ausgangsbasis für Recherchen ideal wären.

* Bernhard Dengg, Mag.iur. Mag.phil., ist Leiter des Bibliotheksbereichs Recht und Wirtschaft der UB Bern. 


\section{A. Bereits bestehende mit SOL-ONline vergleichbare Datenbanken}

In der Schweiz kann man auf zwei Plattformen verweisen, die in ihrer Entstehung Ansätze von Open Access-Plattformen mit sich führten, auch wenn beide auf kommerzieller Ebene erfolgten. Der eine Ansatz ist Swisslex, die wohl grösste und umfangreichste juristische Datenbank der Schweiz, die vor allem von den beiden Verlagen Schulthess und Stämpfli getragen wird und auch zu grossen Teilen deren Verlagsproduktion abdeckt. Die Gründung von Swisslex basierte vor allem auf dem Engagement des Schweizerischen Vereins für Rechtsinformatik, mittlerweile $e$ Justice, im Jahr 1986. Zielsetzung war, eine auf wirtschaftlicher Basis, jedoch kostengünstig geführte digitale Rechtsinformationsversorgung zu gewährleisten. ${ }^{1} \mathrm{Ge}$ rade das Mitwirken aller juristischen Interessensgruppen ist eine wichtige Grundvoraussetzung für den Aufbau einer Open Access Plattform, lebt diese doch vom Austausch und dem Einbringen der einzelnen Kompetenzen.

Den zweiten Ansatz für eine Plattform lieferte die 1999 gegründete Firma Weblaw mit ihrem Internetportal und ihrer Online-Zeitschrift jusletter. In Form eines frei zugänglichen Newsletters sollte die juristische Gemeinschaft rasch und unabhängig vom traditionellen Zeitschriftenwesen über aktuelle Themen informiert werden. Für die Qualitätskontrolle wurden Fachspezialisten gewonnen, die als Redakteure die einzelnen Module betreuen und so für die Qualitätssicherung sorgen. Damit nahm Weblaw den ursprünglichen Grundgedanken des juristischen Zeitschriftenwesens auf, schnell und breit für einen Berufsstand in knappen zeitlichen Abständen zu informieren. Der in den ersten Jahren kostenlos und frei zugängliche jusletter wurde später jedoch kostenpflichtig.

\section{B. Was soll SOL-ONline leisten können?}

SOL-ONline liegt die Idee eines Fachportals zugrunde, das sowohl auf bereits bestehenden Daten als auch auf Erstpublikationen aufbaut. Die Architektur der Plattform sollte in der Lage sein, alle möglichen juristischen Texte aufzunehmen, sowohl Beiträge, als auch Entscheide und Gesetzestexte.

Der Pilot von SOL-ONline basiert auf der Open-Source-Software Samvera. ${ }^{2}$ Das Paket besteht aus Fedora 4 für die Datenspeicherung, sowie Solr für die Indizierung. Beide Programme haben sich bereits für den Aufbau von Repositorien bewährt. Die Software bietet ein offenes und erweiterbares Frontend, welches mit diversen Middleware-Komponenten (unter anderem Blacklight und Solr) ${ }^{3}$ kommuniziert.

1 Ausführlich zur Gründung von Swisslex: Stocker, Werner: «20 Jahre Schweizerischer Verein für Rechtsinformatik SVRI: von den Anfängen bis zur Gegenwart.», Rz 5 ff. in: Jusletter 7. November 2005.

2 Siehe: https://samvera.org/.

3 Siehe: http://projectblacklight.org/; http://lucene.apache.org/solr/. 
SOL-ONline soll die Funktionen eines Meta-Repositories sowie einer Plattform für Direkt- und Zweitveröffentlichungen erfüllen und eine Recherchefunktion sowohl über Metadaten als auch über Volltexte ermöglichen. Suchabfragen zu Gesetzesund Entscheidungssammlungen und das Erstellen von Profilen, Ankündigungen von Veranstaltungen und Stellenausschreibungen runden das Angebot ab.

\section{Meta-Repository}

Alle Schweizer Universitäten betreiben mittlerweile Open-Access-Repositorien, die Metadaten und Volltexte von Publikationen verzeichnen. SOL-ONline importiert und indiziert regelmässig die Metadaten, die bei Einträgen zu juristischen Beiträgen erfasst sind. Damit deckt SOL-ONline auf bibliographischer Ebene die wissenschaftlichen Leistungen an den rechtswissenschaftlichen Fakultäten der Schweiz ab. SOL-ONline setzt zu jedem Eintrag einen Link, so dass der Volltext im jeweiligen institutionellen Repositorium abgerufen werden kann, sofern er dort hochgeladen wurde.

Bei der Einspielung von Metadaten aus den verschiedenen Repositorien wird sich die mangelnde Homogenität und niedrige Granularität der Daten vermutlich als grundlegendes Problem erweisen. Im Gegensatz zu Repositorien werden die Metadaten in Bibliothekskatalogen nach festgelegten internationalen Standards aufgenommen und so miteinander verknüpft, dass bei Recherchen eindeutige Treffermengen erzielt werden können. Dies betrifft unterschiedliche Namensbezeichnungen von Personen, Körperschaften, Geographica, etc., unterschiedliche Titelvarianten, sei es aufgrund Mehrsprachigkeit oder auch Änderungen bei Titeln von Zeitschriften, Schriftenreihen oder Jahrbüchern. Grundsätzlich müssen beim Aufbau eines grossen Metadatenpools bereits von Beginn an die Regeln festgelegt werden, in welcher Weise die Metadaten erfasst und indexiert werden.

Die Erfassung von Metadaten bei Repositorien erfolgt gewöhnlich in drei Schritten. Im ersten Schritt werden die Daten vom Autor oder seinem Institut direkt eingegeben und im besten Fall der Volltext des wissenschaftlichen Beitrages in das Repository hochgeladen. Die eingegebenen Daten werden danach von BibliothekarInnen kontrolliert und korrigiert. Bei hochgeladenen Texten ist zwar der Autor dafür verantwortlich, dass er die Rechteabklärung vorgenommen hat und über die Rechte für eine Zweitveröffentlichung verfügt, jedoch wird zusätzlich von Seiten der Bibliothek eine Abklärung durchgeführt. ${ }^{4}$

4 Da die Editoren nur selten einen Einblick in den Autorenvertrag haben bzw. oftmals keiner abgeschlossen wird, richtet man sich nach den AGBs bei den Verlagen bzw. Zeitschriften und führt über deren Rechtepolitik eine Datenbank. Weiters klärt man die Rechte über die Datenbank SHERPA/ RoMEO (http://www.sherpa.ac.uk/) ab, die die Zweitveröffentlichungsrechtevergabe von Verlagen dokumentiert. 
Als letzter Schritt erfolgt die Freischaltung der Einträge durch die Bibliothek. Da in Repositorien nur wenige Metadaten erhoben werden und diese in den meisten Fällen nur über simple Eingabefelder verfügen, sind die Daten ungenauer als die der Bibliothekskataloge. Diese verfügen zusätzlich über Verweismöglichkeiten und Verknüpfungen zu anderen Daten oder über Instrumente wie Äquivalenzen oder Homonyme. Diese Qualität wird vorerst in SOL-ONline nicht erreicht werden können.

Die Uneinheitlichkeit der Metadaten aufgrund des Zusammenspiels der Daten aus den einzelnen Repositorien wird sich als erhebliches Problem erweisen. Da es bislang noch keine Erfahrungen mit Meta-Repositorien gibt, wird es hier zu grundlegenden Abklärungen kommen müssen. Die Folge davon wird sein, dass einheitliche Standards und Datenformate festgelegt werden müssen. Der Zusammenschluss von Bibliotheken in Verbünden zwecks qualitativer und kostengünstiger Datenpflege könnte den Repositorien dabei als Vorbild dienen. SOL-ONline würde diesen Schritt schon vorwegnehmen.

Auch wenn einzelne Metadaten in den Repositorien mit Volltexten verknüpft sind, werden die Volltexte aus urheberrechtlichen Gründen nicht in SOL-ONline überspielt. Eine Verlinkung zu den Quelldaten sollte jedoch gegeben sein. SOL-ONline wird durch diese Lösung vorerst einen stark bibliographischen Charakter haben, jedoch besteht für die Autoren die Möglichkeit im Zuge einer Zweitveröffentlichung, den Volltext direkt an die bibliographischen Angaben anzuhängen.

\section{Pflege der Metadaten}

Ein entscheidender Faktor für die Qualität einer Datenbank ist die Qualität ihrer Metadaten. Je grösser die Anzahl an Metadaten, desto wichtiger ist es, dass diese nach einheitlichen Regeln bearbeitet werden. Dies betrifft sowohl formelle Eingaben, wie Autor, Titel der Publikation, Titel der Zeitschrift oder des Gesamtwerkes, usw., als auch die inhaltlichen Eingaben wie Schlagwörter oder Klassifikationen.

Im Fall der Eingabe von Publikationen versteht man unter der formellen Erfassung von Daten die einheitliche Beschreibung der Publikation nach festgelegten Kriterien, so dass die Auffindbarkeit der Publikation im Zuge einer Recherche möglich ist. Wie bei Bibliothekskatalogen oder vergleichbaren bibliographischen Datenbanken stellt sich hier auch die Frage der Erschliessungstiefe. Grundsätzlich sollte diese in dem Masse erfolgen, dass eine Publikation individualisierbar ist. Teile einer Publikation werden hingegen nicht eigens erschlossen. Bei der einheitlichen Beschreibung ist darauf zu achten, dass sich wiederholende Eingaben stets nach denselben Kriterien erfasst werden. Hier stellen sich vor allem Fragen bei der Erfassung von Titeln, wie zum Beispiel bei Obertiteln, Parallel- und Nebentiteln sowie Untertiteln, bei Titeln von Zeitschriften oder Schriftenreihen, aber auch bei den 
Namen von AutorInnen, wenn unterschiedliche Schreibweisen oder Abkürzungen verwendet werden. Im Gegensatz zu Bibliothekskatalogen verwendet SOL-ONline vorerst keine Äquivalenzen oder homonyme Zusätze, da dies die Metadatenbetreuung für die zu erwartenden Inhalte ungemein erschweren würde. Hingegen sollte die Metadateneingabe die Verwendung von Abkürzungen bei Zeitschriftentiteln bzw. vorhandener Paralleltitel berücksichtigen können.

Die Eingabe der Daten bei einer Direktpublikation erfolgt im Idealfall vom Autor selbst. Hierzu sollen Indexlisten bei den einzelnen Eingabefeldern die Eingabe erleichtern. Gerade bei Zeitschriftentiteln, Schriftenreihen oder Gesamtwerken soll SOL-ONline bei der Eingabe Vorschläge anbieten können. Zeitschriftentitel können dabei sowohl in ihrer gängigen Abkürzung wie auch als Volltitel eingegeben werden. Für die Eingabe bei den Klassifikationsfeldern und Schlagwörtern erfolgt ein Vorschlag über eine Liste.

Die Kontrolle und Ergänzung der Daten werden von Bibliotheken gewährleistet, die sich bereit erklären, die Pflege zu übernehmen. Diesbezüglich bedarf es eines Verteilschlüssels, der entweder nach regionalen Kriterien oder nach rein formalen Kriterien, wie alphabetische Zuteilung nach Autoren gebildet werden könnte. Diesbezüglich hat es bereits Vorgespräche unter einigen Bibliotheken aus der deutschsprachigen Schweiz gegeben.

\section{E. Rechtsbibliographie}

Durch das Einspielen der Daten aus den einzelnen Repositorien der Schweizer Universitäten erfüllt SOL-ONline auch den Zweck einer, wenn auch nicht umfassenden, Rechtsbibliographie. Eine Schweizer Rechtsbibliographie wurde von 1945 bis 2004 als Printausgabe geführt und ab 2005 als Datenbank vom Stämpfli Verlag auf uni.recht.ch. Auch wenn eine Rechtsbibliographie keine Volltexte aufweist, so ist sie immerhin ein wichtiger Leistungsausweis der juristischen Publikationstätigkeit. Möglich wäre auch die Einspielung von Metadaten aus Bibliothekskatalogen, so dass selbstständige Werke wie Monografien, Kommentare, Fest- und Kongressschriften aufgeführt sind. Ein juristischer Metakatalog besteht bereits mit jusbib. ${ }^{5}$ Dieser fasst die Bestände aller wichtigen juristischen Bibliotheken der Schweiz zusammen, auch auf Artikelebene. Ab dem Jahr 2021 wird durch SLSP (Swiss Library Service Plattform) zudem ein schweizweiter Verbundkatalog zur Verfügung stehen, aus dem auch die Metadaten von juristischen Publikationen eingespielt werden könnten. Wie umfassend hier die Erfassungstiefe sein wird, ist jedoch noch offen.

5 https://jus.swissbib.ch/. 


\section{F. Publikation von Volltexten}

SOL-ONline bietet auch die Möglichkeit Volltexte zu veröffentlichen. Dabei können Texte im Zuge einer Zweitpublikation, aber auch als Direktpublikation deponiert werden. Werden die Texte als Erstpublikation hochgeladen, so sind diese von einer Redaktion zu genehmigen, um die Qualität der in SOL-ONline vorhandenen Texte zu gewährleisten. Dieser Open-Access-Aspekt ist das zentrale Element von SOL-ONline. Vorrangig wird SOL-ONline auf die Publikation auf Artikelebene ausgerichtet sein, aber auch die Einspielung von Volltexten grösseren Umfangs wie bei Monografien, insbesondere Dissertationen, wird möglich sein.

SOL-ONline wird zwar in der Lage sein, Zeitschriften zu hosten und diese als Titel zu führen, der strukturelle Aufbau für eine inhaltliche Recherche sollte jedoch in Fachmodulen erfolgen. Eine grundsätzliche Frage wird die Gewährleistung der Qualitätssicherung sein. Hierzu wird SOL-ONline auf die Mitarbeit von Fachexperten angewiesen sein, die die einzelnen Module redaktionell betreuen und das Peer-Review-Prozess gewährleisten.

\section{G. Quellenmaterialien}

Eine juristische Plattform sollte über das notwendige Quellenmaterial verfügen. Auch wenn diese bereits auf anderen offen zugänglichen Plattformen wie zum Beispiel bei Gesetzessammlungen in lexfind.ch oder Bundesgerichtsentscheide auf bger.ch bequem zu recherchieren sind, so ist der schnelle Zugriff auf Normen und Entscheide ein wesentlicher Faktor für die Nutzungsintensität einer Datenbank. Hinzu kommt, dass auch Quellenmaterialien in die personalisierten Literaturlisten aufgenommen werden können.

Im besten Fall bietet SOL-ONline in den vorhandenen Volltexten Verlinkungen zu den einzelnen Quellen an. Wie gross der technische Aufwand für die Erstellung von Verlinkungen sein wird, ist jedoch noch offen und wird in der Umsetzung vorerst nicht prioritär verfolgt.

\section{H. Benutzerprofile und Tools von öffentlichem Interesse}

Durch die Erstellung von Benutzerkonten sollten Juristen auch die Möglichkeit erhalten, ihr Profil zu veröffentlichen, unabhängig davon, ob sie eine Publikation hochladen. Von Interesse wären dabei neben dem beruflichen Profil auch, Fachschwerpunkte, beruflicher Werdegang, Publikationslisten, Vortragstätigkeit, etc. SOL-ONline würde somit in einem gewissen Ausmass eine Funktion wie bei Anbietern wie LinkedIn oder XING erfüllen, die es ermöglicht, sich untereinander besser zu vernetzen, aber auch, sich einer Öffentlichkeit zu präsentieren. 
Eine weitere Funktion im Zusammenhang mit Benutzerprofilen, wäre die Erstellung von Literaturlisten, so wie sie in den gängigen Literaturverwaltungsprogrammen üblich sind. Mit der Möglichkeit diese zu teilen, könnten Lehrende zum Beispiel eine Art «Semesterapparat» für ihre Studierenden erstellen oder Anwaltskanzleien sich ihre virtuelle Bibliothek aufbauen oder die Metadaten mit ihrer faktischen Bibliothek verknüpfen.

Von weiterem öffentlichen Interesse dürfte auch das Angebot für Stellenanzeigen und Veranstaltungshinweisen sein. Zwar gehören diese Angebote nicht zu den Kernaufgaben einer Datenbank, doch erhöhen diese den Anreiz, SOL-ONline aufzusuchen und als zentrale Plattform für die Interessen der juristischen Gemeinschaft zu nutzen.

\section{Probleme, Gefahren, Risiken}

Wie anfangs ausgeführt ist SOL-ONline ein Projekt, das die Möglichkeiten einer alternativen Publikationsplattform ausloten will. Die UB Bern als Projektinitiatorin beabsichtigt nach der Erstellung des Pilotes von SOL-ONline nicht, dieses zu einem fertigen Produkt auszubauen und als verantwortliche Institution zu betreiben. Für den Fall, dass SOL-ONline auf Akzeptanz stösst muss eine Trägerschaft gefunden oder gebildet werden, die bereit ist, SOL-ONline zu einem nachhaltigen Produkt zu entwickeln und eine Finanzierung zu gewährleisten.

Die zu erwartenden Kosten werden hauptsächlich Personalkosten sein. Neben dem Aufbau und der Pflege der technischen Infrastruktur bedarf es auch noch einer Stelle für eine Geschäftsführung, die vor allem den Kontakt zu den begleitenden Gremien schafft und für den Redaktionsstab hinsichtlich der Qualitätssicherung sorgt.

Inhaltlich wird sich SOL-ONline auch mit der Pflege der Metadatenqualität auseinandersetzen müssen. Hier wären die einzelnen Fachbibliotheken, die bereits mit der Pflege der Bibliothekskataloge und Repositorien betraut sind, die geeigneten Ansprechpartner. Dass die Schweizer Rechtsbibliotheken sich schon mehrmals ausserhalb ihrer Kernaufgaben für bundesweite Projekte engagiert haben, sieht man an der Pflege der Schweizer Rechtsbibliographie, deren Eingaben die Bibliotheken im Rahmen der Vereinigung der juristischen Bibliotheken der Schweiz (VJBS) aufgestellt haben.

Zum Schluss sei angeführt, dass sich das Projekt SOL-ONline nicht als Konkurrenzprodukt zum traditionellen Publikationsmarkt sieht, sondern vielmehr als Ergänzung und als Ideengeber für dessen Weiterentwicklung. Das juristische Publikationswesen ist traditionell auf Printmedien ausgerichtet. Dass dies auch zukünftig so sein wird, beweist die nun schon seit längerem bestehende Koexistenz von Da- 
tenbanken und Printwesen. SOL-ONline soll dabei unterstützend für ein diversifiziertes Publikationswesen und für eine Informationsversorgung ausserhalb der kommerziellen Verwertung wissenschaftlicher Publikationen stehen. 